\title{
How reliable are the current Blood Pressure Measuring devices in Health Facilities of
}

\section{Bhutan?}

\author{
Soira S. Tamang ${ }^{1}$, Mongal S. Gurung ${ }^{2}$, Tshewang Lhaden³, Tashi Penjore ${ }^{4}$, Karma Tenzin ${ }^{5}$ \\ ${ }^{1,3,4}$ Department of Medical Supplies and Health Infrastructure, Ministry of Health, Thimphu, Bhutan \\ ${ }^{2}$ HMIS and Research Section, Policy and Planning Division, Ministry of Health, Thimphu, Bhutan \\ ${ }^{5}$ Faculty of Postgraduate Medicine, Khesar Gyalpo University of Medical Science of Bhutan, Thimphu, Bhutan
}

\begin{abstract}
Background: Accurate blood pressure measurement is vital before any inferences are made on the reading. Since device error is one of the potential causes of inaccurate results, blood pressure measuring devices should be periodically assessed for its accuracy. This paper describes the types of devices used in health facilities of Bhutan and the results of their performance verifications. Methods: This study assessed the pressure accuracy and leak rates of blood pressure measuring devices in use at two regional referral hospitals, district hospitals and Basic Health Unit I using calibrated Vital Signs Simulator (ProSim ${ }^{\mathrm{TM}} 8$ ) from Fluke Biomedical as reference device. For pressure accuracy assessment, static pressures were simulated against which simultaneous readings of each of the devices were recorded $(n=3)$. For the leak rate assessment, the simulator was set to a target pressure and leak rate $(n=3)$ were recorded over 1 minute. Results: A total of 395 devices of three types, viz., 135 aneroid, 125 electronic and 135 mercury were assessed. Deviations in readings of $64.72 \%$ of devices were found to be within acceptable range of $\pm 3 \mathrm{mmHg}$. The device-type specific pass percentages for pressure accuracy were $6.40 \%$ for electronic, $88.81 \%$ for aneroid and $94.81 \%$ for mercury. A total of $71.85 \%$ of devices (aneroid and mercury) had acceptable leak rates of $\leq 15 \mathrm{mmHg}$ per minute. Conclusion: The study shows that not all the blood pressure measuring devices currently being used in the health facilities of Bhutan are accurate. Besides ensuring that only validated blood pressure measuring devices enter the country, these devices should be verified for its performance periodically once they are in use.
\end{abstract}

Keywords: Accuracy; Blood Pressure; Devices; leak; ProSim8 simulator.

\section{INTRODUCTION}

Blood pressure measuring (BPM) devices is basic yet fundamental tool in routine clinical care. It measures blood pressure, which is of utmost importance in diagnosis and management of hypertension ${ }^{1-3}$. It is crucial to ensure that the blood pressures measured are accurate before any inferences are made ${ }^{4}$. Studies have reported that either over or under estimation of blood pressure by 3-5 $\mathrm{mmHg}$ could classify 'non-hypertensive' patients as hypertensive and vice-versa ${ }^{3,5,6}$.

Inaccurate devices are potentially the fundamental source for erroneous measurement $t^{4,7}$. Further, with the mercury devices (gold standard) being progressively banned, there has been rapid advancement in technology to replace these devices ${ }^{8,9}$.

Corresponding author:

Soira S. Tamang

sstamang@health.gov.bt
This increases the quest for reliable devices.

Ideally, BPM devices are validated as per credible protocols developed by recognized institutions like the Association for the Advancement of Medical Instrumentation, the British Hypertension Society and the European Society of Hypertension. However, only few manufacturers have published evidences of validation 9 .

This necessitates internal mechanism of assessing the performance of the devices, especially in a country like Bhutan, where medical equipment are still being imported. The Ministry of Health recently endorsed the standard operating procedure on performance verification and safety testing of BPM devices. It recommends the device pressure reading to be within $+/-3 \mathrm{mmHg}$ and leak rate $<=15 \mathrm{mmHg} / \mathrm{min}$. However, the performances of BPM devises that are currently being used in Bhutan were not assessed. Therefore, this study was undertaken with the aim of describing the types of blood pressure measuring devices used in the health facilities in Bhutan and to assess their accuracies. 


\section{METHODS}

\section{Design}

This descriptive cross-sectional study was conducted from May to August 2018.

\section{Setting}

Bhutan is a small landlocked South Asian country in the heart of the Himalayas with a population of 735553 . There are three referral hospitals, 32 hospitals including the traditional medicine hospital and five other hospitals, 23 Basic Health Unit Is, 185 Basic Health Unit IIs, 59 Indigenous units, 45 Sub-posts and 551 Out-Reach Clinics in Bhutan ${ }^{10}$.

\section{Sample population and variables}

For this study, two referral hospitals (Central and Eastern Regional Referral Hospital), all hospitals and Basic Health Units Grade I were included. The study was not performed at BHU IIs, Sub-posts, ORCs, and hospitals managed by military due to time and budgetary constraints.

All functional BPM devices (mercury, aneroid and electronic) within the aforementioned health facilities were assessed for pressure accuracy and leak rates. Physical conditions of the devices were checked before the commencement of tests. Defective devices were repaired on site (replacement of cuffs, bladders, inflating bulbs, metal valves, glass graduated tube, connectors, and electronic components). For aneroid BPM devices, the baseline/pointer was re-adjusted to $0 \mathrm{mmHg}$, wherever necessary. Similarly, for mercury BPM devices, the level of mercury was maintained at $0 \mathrm{mmHg}$.

Calibrated Vital Signs Simulator (ProSim ${ }^{\mathrm{TM}}$ 8) from Fluke Biomedical was used as the reference device. It had measurement error of $\pm 0.016 \mathrm{mmHg}$ and uncertainty of \pm 0.58 $\mathrm{mmHg}$ (at 95\% confidence interval) over the pressure range 10$400 \mathrm{mmHg}$. The simulator was calibrated at Tektronix Private Limited, Mumbai, India before the commencement of the study. The 'NIBP' function of the reference device was selected for this study.

\section{Set up}

The BPM devices under test (DUT) were connected to the reference device using a Tee connector as shown in Figure 1. The third open-end of Tee connecter was connected with one of the ends of cuff. The cuff was wrapped around a $9 \mathrm{~cm}$ mandrel. For aneroid and mercury BPM devices, the metal/bleed/air release valves of inflating bulb (other end of cuff) were tightly secured. Both DUT and reference device were placed on flat surface before the tests.

\section{Pressure Accuracy Test}

'PRESSURE SOURCE' test mode was selected for this test. Residual pressures on the DUT and reference device were set to zero. DUT were exposed to static pressures simulated by ProSim8 and simultaneous readings on DUT were recorded. Three readings were taken for each of the simulation. For electronic BPM devices, pressures (systolic/diastolic) were simulated at; i) $180 / 130 \mathrm{mmHg}$ ii) $80 / 40 \mathrm{mmHg}$ iii) $120 / 80 \mathrm{mmHg}$, pseudo 'hyper', 'hypo' and 'normo' tensive conditions respectively. The sequence was maintained to avoid possible errors introduced by DUT's (electronic devices) temporary memory. For mercury and aneroid devices, pressures were simulated at; i) $150 \mathrm{mmHg}$ ii) 80 $\mathrm{mmHg}$ iii) $100 \mathrm{mmHg}$.

\section{Leak Test}

The 'LEAK TEST' mode on the reference device was selected for this test. First the leak test was performed on the reference device without connecting DUT to offset the leak rate of the whole system. The leak tests could not be performed on electronic devices, as it could not be set to 'calibration mode', which was a pre-requisite. Three $(n=3)$ readings were taken at the simulated target pressure of $150 \mathrm{mmHg}$ over 1 minute.

\section{Data Analysis and Ethical Approval}

Data was double entered in EpiData (version 3.1), validated and transferred to Stata version 13.1 for analysis. For this study descriptive statistics such as frequency and proportion were calculated. For pressure accuracy test, differences between the simulated and averaged corresponding recorded pressure readings were taken. The DUT with differences within $\pm 3 \mathrm{mmHg}$ were considered accurate. For leak test, DUT with average leak rate $\leq 15 \mathrm{mmHg}$ were considered acceptable.

Since there was no human subject involvement in the study the Research Ethics Board of Health exempted the study from ethical review. The administrative clearance was obtained from Ministry of Health prior to commencement of this study.

\section{RESULTS}

All 395 functional blood pressure measuring devices, being used at the study sites, were tested in this study. These devices were of three types; aneroid (34.18\%), electronic (31.65\%) and the remaining were mercury devices as shown in Table 1.

Out of 394 devices tested for pressure accuracy, $64.72 \%$ passed the pressure accuracy test i.e. the readings were within the acceptable range of $\pm 3 \mathrm{mmHg}$ as shown in Table 2. One aneroid device could not be tested for pressure accuracy as the leakage rate was above $80 \mathrm{mmHg}$, which did not allow static pressure reading. In terms of type of devices, $6.40 \%$ of electronic devices passed the pressure accuracy tests as compared to $88.81 \%$ of aneroid and $94.81 \%$ of mercury devices. Leak test was performed only for mercury and aneroid devices due to technical limitations. A box plot of leak rates for these devices is shown in Figure 2. Out of 269 devises tested, $71.85 \%$ passed the test with readings within the national allowable leak rate of $\leq 15 \mathrm{mmHg}$ per min.

The devices manufacturers were Omron $(22.53 \%)$, Diamond (21.27\%), Rossmax (18.48\%), Lifeline (6.33\%), Dr. 
Table 1. Distribution by type, manufacturer and model of Blood Pressure (BP) measuring devices in health facilities in Bhutan, 2018

\begin{tabular}{|c|c|c|c|c|}
\hline \multirow[b]{2}{*}{ Description of devices } & \multicolumn{4}{|c|}{ Health Facilities } \\
\hline & $\begin{array}{c}\text { Referral Hospital } \\
\boldsymbol{n}(\%)^{*}\end{array}$ & $\begin{array}{c}\text { District Hospital } \\
n(\%)^{*}\end{array}$ & $\begin{array}{c}\text { BHU I } \\
n(\%)^{*}\end{array}$ & $\begin{array}{l}\text { Total } \\
n(\%)^{*}\end{array}$ \\
\hline \multicolumn{5}{|l|}{ Type } \\
\hline Aneroid & $16(25.40)$ & $62(29.67)$ & $57(46.34)$ & $135(34.18)$ \\
\hline Electronic & $20(31.75)$ & $72(34.45)$ & $33(26.83)$ & $125(31.65)$ \\
\hline Mercury & $27(42.85)$ & $75(35.88)$ & $33(26.83)$ & $135(34.17)$ \\
\hline Total & 63 & 209 & 123 & 395 \\
\hline \multicolumn{5}{|l|}{ Manufacturer } \\
\hline Omron & $11(17.46)$ & $48(22.97)$ & $30(24.39)$ & $89(22.53)$ \\
\hline Diamond & $22(34.92)$ & $44(21.05)$ & $18(14.63)$ & $84(21.27)$ \\
\hline Rossmax & $11(17.46)$ & $33(15.79)$ & $29(23.58)$ & $73(18.48)$ \\
\hline Lifeline & $2(03.17)$ & $15(07.18)$ & $8(06.50)$ & $25(06.33)$ \\
\hline Dr.Sphygmomanometer & $1(01.59)$ & $10(04.78)$ & $9(07.32)$ & $20(05.06)$ \\
\hline Others $^{\dagger}$ & $16(25.40)$ & $56(26.79)$ & $28(22.76)$ & $100(25.32)$ \\
\hline Missing & $0(0.00)$ & $3(01.44)$ & $1(00.81)$ & $4(01.01)$ \\
\hline \multicolumn{5}{|l|}{ Model } \\
\hline \multicolumn{5}{|l|}{$(\text { Rossmax })^{\S}$} \\
\hline GB101 & $8(12.70)$ & $30(14.35)$ & $27(21.95)$ & $65(16.46)$ \\
\hline \multicolumn{5}{|l|}{$(\text { Diamond })^{\|}$} \\
\hline Regular (CM/L-0196043) & $20(31.75)$ & $38(18.18)$ & $13(10.57)$ & $71(17.97)$ \\
\hline \multicolumn{5}{|l|}{ (Omron) } \\
\hline HEM7117 & $11(17.46)$ & $24(11.48)$ & $22(17.89)$ & $57(14.43)$ \\
\hline Others $^{* *}$ & $7(11.11)$ & $61(29.19)$ & $21(17.07)$ & $89(22.53)$ \\
\hline Missing $^{\dagger \dagger}$ & $17(26.98)$ & $56(26.79)$ & $40(32.52)$ & $113(28.61)$ \\
\hline
\end{tabular}

${ }^{*}$ Column percentages

$\dagger$ Includes Citzen, Gold Supreme,Dr.Morepen,Hicks,Bookang, Medifit, Accusure, Naulakha, Kenzin, Equinox, ALP K2, MSI, Bio Plus, Microlife, Boso, Riesta, A\&D, Tycos, SK, Focal, Extra care. The manufacturers $<5 \%$ were clubbed under others.

Devices whose detail of manufacturers were missing

$\S$ Aneroid BP measuring Devices

"Mercury BP measuring Devices

"Electronic BP measuring Devices

**Includes HEM-8712, HEM-4500-SOL, Deluxe, CM/L 9421877, CM/L 8325472, Exacta, CL0483, CM/L 8262373, CH-432, BP Fit Pro, HEM-7120, HEM-8712, BP-09, GB102, HEM-71111, HEM-7121, D-72417, CH-453-AC, CH-463E, BP3AQI-2P, HEM7200-C1, KI, BP-05. The models $<5 \%$ were clubbed under others.

†Devices whose detail of models were unavailable 
Table 2. Pressure Accuracy test results of various blood pressure measuring devices in health facilities of Bhutan with ProSim8, 2018

\begin{tabular}{lcccc}
\hline Type & Total tested & $\begin{array}{c} \pm \mathbf{3} \mathbf{~ m m H g} \\
\boldsymbol{n}(\mathbf{\%})\end{array}$ & $\begin{array}{c} \pm \mathbf{5} \mathbf{~ m m H g} \\
\boldsymbol{n}(\mathbf{\%})\end{array}$ & $\begin{array}{c} \pm \mathbf{1 0} \mathbf{~ m m H g} \\
\boldsymbol{n}(\mathbf{\%})\end{array}$ \\
\hline Mercury & 135 & $128(94.81)$ & $130(96.30)$ & $133(98.52)$ \\
Aneroid & 134 & $119(88.81)$ & $123(91.79)$ & $131(97.76)$ \\
Electronic & 125 & $8(6.40)$ & $26(20.80)$ & $113(90.40)$ \\
Overall & 394 & $255(64.72)$ & $279(70.81)$ & $377(95.68)$ \\
\hline
\end{tabular}

"Unable to read pressure for one device as the leak rate was too high to maintain static pressure

${ }^{\dagger}$ The permissible range as specified in the standard operating procedure for performance verification and safety testing of blood pressure approved by the Ministry of Health for use in Bhutan

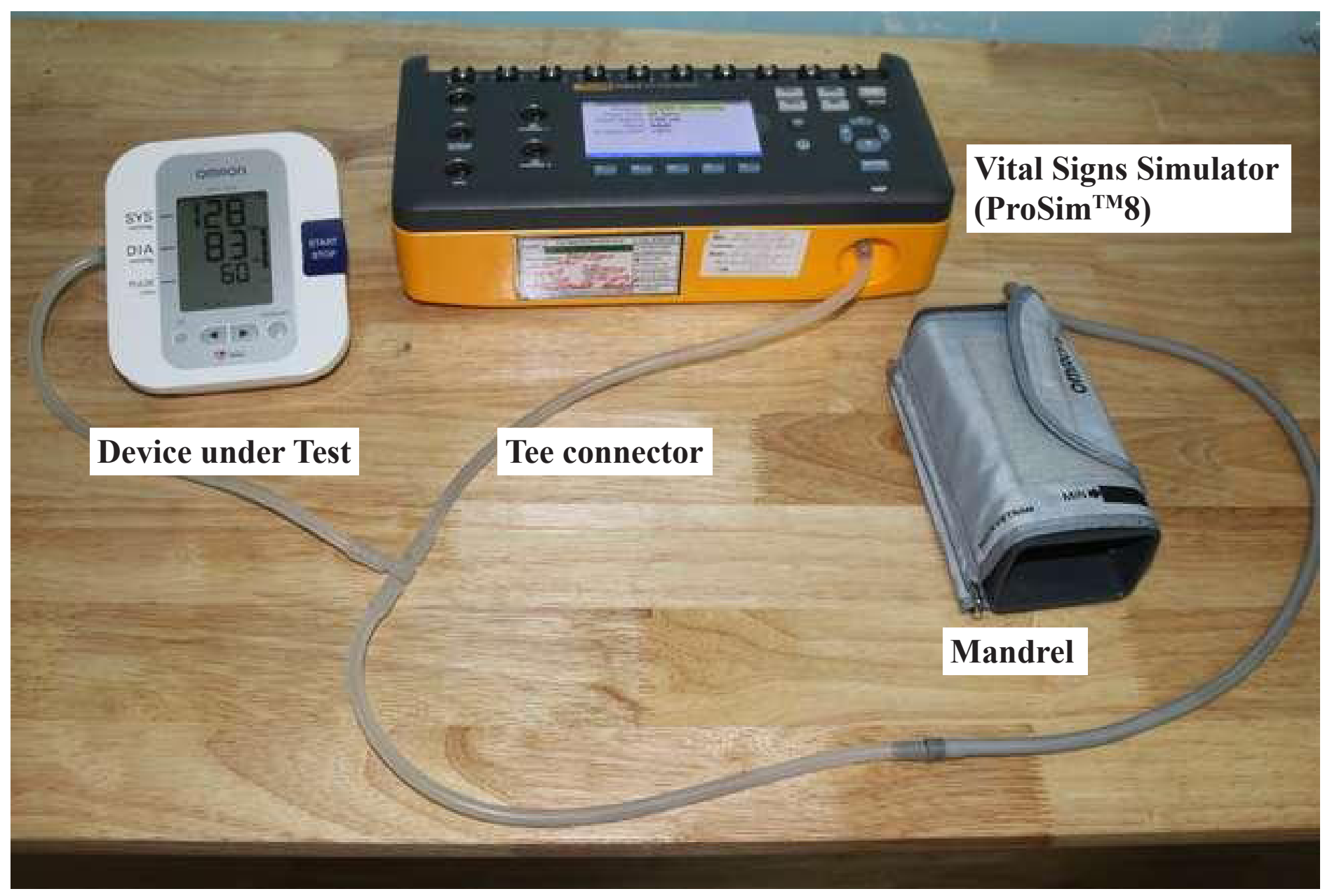

Figure 1. Set up of Blood Pressure Measuring Device for Pulse accuracy and leak tests with Vital Signs Simulator (ProSimTM8), in health facilities of Bhutan, 2018 as per the standard operating procedure for performance verification and safety testing of blood pressure approved by the Ministry of Health for use in Bhutan 


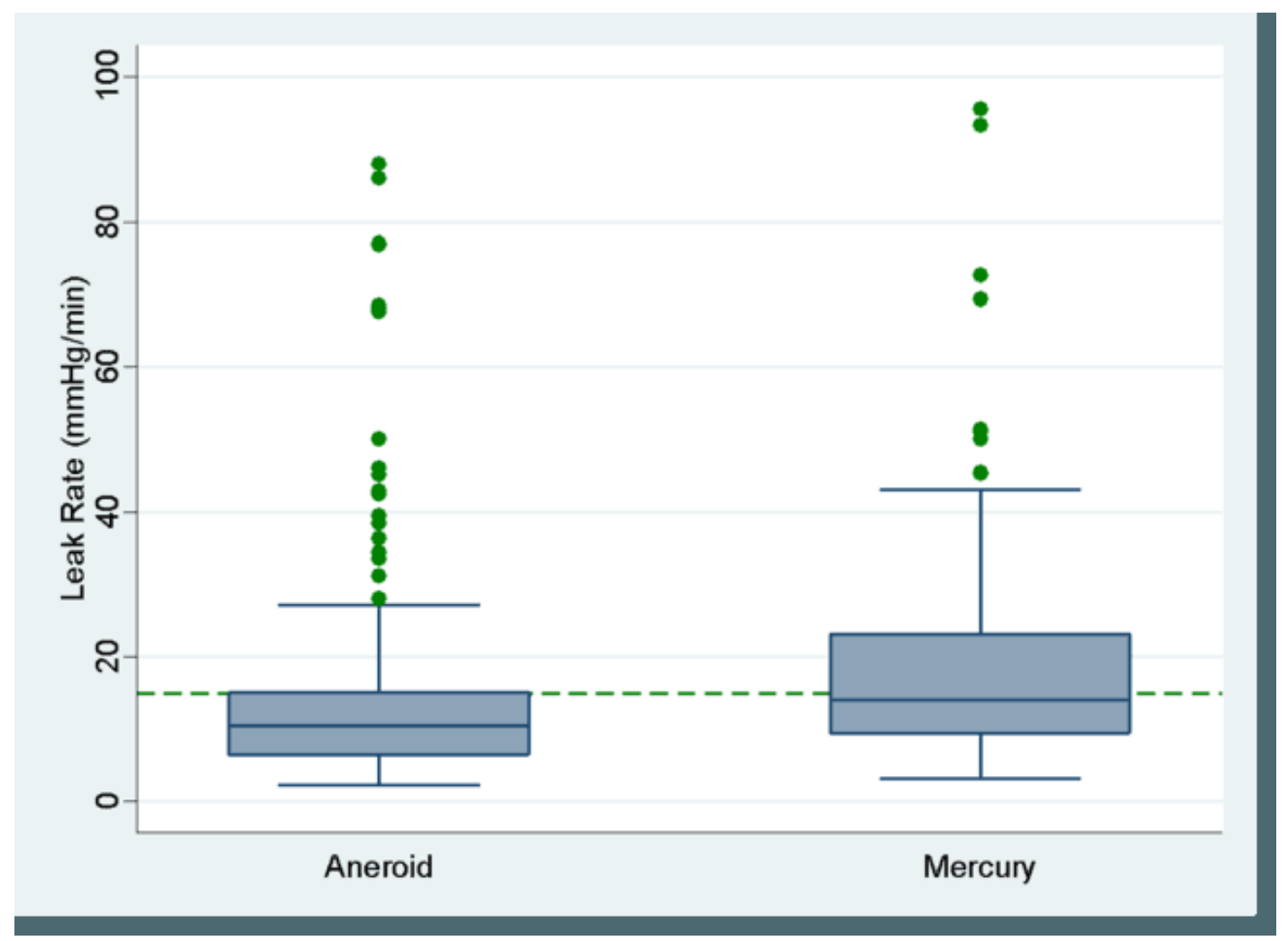

Figure 2. Box plots of the measured leakage rate in aneroid and mercury blood pressure measuring devices $(n=269)$ in health facilities of Bhutan, 2018. Dash line indicate the permissible leakage rate $(<=15 \mathrm{mmHg})$ as specified in the standard operating procedure for performance verification and safety testing of blood pressure approved by the Ministry of Health for use in Bhutan

Sphygmomanometer $(5.06 \%)$ and others $(25.32 \%)$. Out of 282 devices for which the data on type of models were available, 14.43\% were HEM-7117 from Omron, $16.46 \%$ were GB101 from Rossmax and 17.97\% CM/L-0196043 from Diamond (Table 1). Other models, which were less than $5 \%$ by individual model, constituted $22.53 \%$.

\section{DISCUSSION}

This study found that two-thirds of the BPM devices used in the selected health facilities of Bhutan in 2018 were accurate to \pm 3 $\mathrm{mmHg}$. However, more than ninety percent of electronic devices were found to be inaccurate. This finding is worrisome for Bhutan where there is a gradual phasing out of mercury devices and ever increasing use of electronic devices, both at health facilities and homes.

Inaccurate reading of BPM devices could mean risks of 'overtreatment' or 'under treatment' ${ }^{1,11}$. This may lead to depriving needy patients of anti-hypertensive drugs, unwanted psychological distress and unnecessary expenditure on drugs? On the other hand accurate measurements mean early detection of hypertension, thus, efficient management of patients cutting down country's expenditure on drugs and possibly referrals. Although the studies on the performances of BPM devices from the developing countries are scanty, a study conducted at St. Thomas' Hospital, United Kingdom (UK), reported that only 26 percent of electronic BPM devices were inaccurate ${ }^{9}$. The better result in that study could be because of the better model of electronic devices used in the UK. In another study, Stergiou et al conducted a review of 28 articles in 2017 which included 31 validation studies on 29 devices reported that $22(71 \%)$ devices passed, while $9(29 \%)$ of them failed the BP measuring protocols ${ }^{8}$. The difference in results could be again due to variation in: the model of devices tested, and validation protocol.

The study from a hospital in the UK has reported 13 out of the 47 electronic BPM devices tested to have published evidence of validation'. However, almost all the BPM devices used in health facilities of Bhutan do not have published evidence of validation. Although, device models such as $\mathrm{CH}-432 \mathrm{~B}$, HEM7117-E, HEM-4500-SOLE, HEM-7200-E had evidence of validation (dableducational website), these are recommended for home use and not for clinical use. Further, there are no standards and guidelines for the import of BPM devices in the country. 
It is also clinically important to know air leakage rates as it assesses the condition of the cuff, rubber bag, rubber tubing, connections and metal/bleed valves. The Leak test results illustrates that over two thirds of devices qualifying the tests with leak rates within national acceptable range of $<=15 \mathrm{mmHg}$. A study reported as low as only three percent of devices qualifying the test ${ }^{9}$. This could be due to the stringent permissible $(<=4$ $\mathrm{mmHg} / \mathrm{min}$ ) leak rate as per European standard. However, the study considered a threshold of $60 \mathrm{mmHg} / \mathrm{min}$ above which, the leakage would cause inaccurate measurement. Comparing to the mercury devices, aneroid devices performed better in terms of leak test. This is also reported to be true in the study mentioned earlier9.

\section{Strengths}

This is the first study highlighting the accuracy assessment of BPM devices in selected health facilities of Bhutan. Since the study was conducted on two referral hospitals, all hospitals and all Basic Health Unit (BHU I), the findings can be representative of national level. It can also serve as a baseline for future related studies, which will ensure the quality of blood pressure measuring devices to be purchased and provided to health facilities in the country.

The methodology adopted could provide an alternative for validation of BPM devices during use. Else the validation protocols are not feasible in a routine clinical care because the procedures are lengthy, time consuming, and have restrictive selection criteria for recruitment of subjects.

The study used a calibrated reference device, which applied static pressures to mandrel rather than employing human subjects, whose blood pressures can vary depending on circumstance or environment.

\section{LIMITATIONS}

The age of the individual BPM devices could not be obtained. Thus the study could not comment on likely impact of the age of devices on their accuracy. The sensor and pressure transducer in electronic devices are reported to be losing their stability with time 9 .

Pulse accuracy measurements were not recorded for all of the electronic BPM devices, therefore not reported. Further, these devices could not be set to calibration mode, thus, leak tests were also not performed.

\section{RECOMMENDATIONS}

As an immediate measure, health centers were notified and advised to adjust respective differences in reading of their respective BPM devices. Those devices with erratic readings were condemned on spot. Whilst, temporary administrative actions were taken based on the findings, this calls for standardizing the models of the devices being purchased. Care and efforts should be taken to ensure that validated devices are imported into the country.
However, a recent study states the possibility of manufacturer's influence on validation outcome especially those who sponsor the validation ${ }^{1}$. Thus, BPM devices need to be re-validated with internally developed procedures at par with the recognized protocols. Further, their performances need to be checked and verified periodically to ensure their accuracy.

The study findings also raise concerns on devices purchased by the public, for home use, from the local retailers in the country. There are no regulatory controls to monitor such purchases. Whilst, blood pressures are measured in the clinical set up before making any inferences, chances are people can rely on faulty readings and avoid their visits to clinics if found low.

\section{CONCLUSIONS}

The study underscores that almost all electronic blood pressure measuring devices that are currently being used in the health facilities of Bhutan are not within the national accuracy range. Therefore, measures to ensure that only validated devices are imported in the country needs to be strengthened. Further, BPM devices should be verified for its performance periodically once they are in use.

\section{ACKNOWLEDGMENTS}

This research was conducted as part of Bhutan's Structured and Mentoring Approach to Research Training (B SMART) course, offered by the Khesar Gyalpo University of Medical Sciences of Bhutan in collaboration with the Ministry of Health, Bhutan, with financial support from the World Health Organization, Bhutan.

\section{REFERENCES}

1. Pickering TG, Hall JE, Appel LJ, Falkner BE, Graves J, et al. Recommendations for blood pressure measurement in humans and experimental animals: Part 1: Blood pressure measurement in humans - a statement for professionals from the subcommittee of professional and public education of the American Heart Association Co. Circulation. 2005;111(5):697-716. [Full Text]

2. O'Brien E, Asmar R, Beilin L, Imai Y, Mancia G, Mengden $\mathrm{T}$, et al. Practice guidelines of the European society of hypertension for clinic, ambulatory and self blood pressure measurement. J Hypertens. 2005;23:697-701. [Full Text $\mathrm{DOI}]$

3. Zuber M, Sch H, Kaiser W, Erne P. Measuring accuracy of sphygmomanometers in the medical practices of Swiss primary care physicians. 2012 April:244-7. [Full Text | DOI]

4. O'Brien E, Stergiou GS. The quest for accuracy of blood pressure measuring devices. 2018 March:2015-8. [Full Text | DOI] 
5. Cleland MJ, Pham B, Miller DR. Equipment: Influence of arrhythmias on accuracy of non-invasive blood pressure monitors. Can J Anaesth 1998; 45(7): 699-705. [Full Text | DOI]

6. Boubouchairopoulou N, Kollias A, Atkins N, O’Brien E, Stergiou GS. Validation of blood pressure monitors using the AMMI and ISO protocols. Journal of Hypertension. September 2016;34:669-705. [Full Text | DOI]

7. Handler J. The importance of accurate blood pressure measurement. Perm J [Internet]. 2009;13(3):51-4. [PubMed | Full Text | DOI]

8. Choi S, Kim Y, Shin J, Lim Y, Choi S, et al. Comparison of the accuracy and errors of blood pressure measured by 2 types of non-mercury sphygmomanometers in an epidemiological survey. 2018;25. [PubMed | Full Text | DOI]
9. Greeff A, Lorde I, wilton A, Seed P, Coleman AJ, shennan AH. Calibration accuracy of Hospital-based non-invasive blood pressure measuring devices. J Hum Hypertens. 2010;24:58-63. [Full Text | DOI]

10. Ministry of Health. Annual Health Bulletin 2017. Thimphu, Bhutan: Policy \& Planning Division, Minintry of Health; 2017.123p. [Full Text]

11. Turner MJ, Baker AB, Kam PC. Effects of systematic errors in blood pressure measurements on the diagnosis of hypertension. Blood Press Monit. 2004;9(5):249-53. [Full Text]

\section{AUTHORS CONTRIBUTION}

Following authors have made substantial contributions to the manuscript as under:

SST: Concept, analysis of data, study design, manuscript drafting and critical reviews

MSG: Analysis of data, study design, manuscript revision

TL: Analysis of data, study design, data collection

TP: Study design, manuscript drafting and revision

KT: Analysis of data, manuscript drafting and revision

Authors agree to be accountable for all respects of the work in ensuring that questions related to the accuracy and integrity of any part of the work are appropriately investigated and resolved.

\section{CONFLICT OF INTEREST \\ None \\ GRANT SUPPORT AND FINANCIAL DISCLOSURE \\ None}

